\title{
INFLUÊNCIA DO TEMPO DE REAÇÃO NA PRODUÇÃO DE BIODIESEL VIA CATÁLISE HETEROGÊNEA
}

\author{
J. S. A. CINTRA ${ }^{*}$, G. A. G. PEDROZA, M. N. PORTELA, T. C. de C. SILVANY, L. C. L. SANTOS, A. K. C. L. LOBATO \\ Programa de Pós-Graduação em Engenharia Química, Universidade Federal da Bahia (PPEQ/UFBA) \\ jackeline.cintra@yahoo.com ${ }^{*}$
}

Submetido 10/10/2016 - Aceito 01/02/2017

DOI: $10.15628 /$ holos.2017.5197

\section{RESUMO}

O biodiesel apresenta muitas vantagens em comparação ao diesel por ser de origem renovável, biodegradável, podendo ser empregado de forma ambientalmente sustentável. A sua produção é comumente realizada através da reação de transesterificação por catálise homogênea. Entretanto, os catalisadores heterogêneos têm sido amplamente estudados por serem eficientes e passíveis de reciclagem, além de facilitar a purificação do biodiesel e minimizar a geração de efluentes. Neste trabalho, o óxido de cálcio ( $\mathrm{CaO}$ ) foi escolhido como catalisador básico para a reação de transesterificação por apresentar maior atividade, condições suaves de reação, menor custo e baixa toxidade, quando comparado com outros catalisadores comerciais. Foram avaliados os efeitos do tempo de reação no rendimento do biodiesel produzido com óleo de soja via rota metílica num processo em batelada, fixando a razão molar metanol/óleo em $12: 1$, temperatura em $65^{\circ} \mathrm{C}$ e concentração de catalisador em $8 \%$ em peso do óleo e variando o tempo do processo em $1 \mathrm{~h}, 1,5 \mathrm{~h}$ e $2 \mathrm{~h}$. Verificou-se que os efeitos relativos à variação do tempo de reação utilizada na síntese de biodiesel influenciam diretamente no processo.

PALAVRAS-CHAVE: Biodiesel, Transesterificação, Catalisador Heterogêneo.

\section{INFLUENCE OF REACTION TIME IN BIODIESEL PRODUCTION VIA HETEROGENEOUS CATALYSIS}

\begin{abstract}
Biodiesel has several advantages compared to diesel, since it is produced from renewable sources, it is biodegradable and can be used in an environmentally sustainable way. Its production is commonly carried out through transesterification by homogeneous catalysis. However, heterogeneous catalysts have been widely studied due to its efficiency and for being suitable for reuse. In addition, heterogeneous catalysts could facilitate the purification of biodiesel and also to minimize the generation of waste. In this work, calcium oxide ( $\mathrm{CaO}$ ) was chosen as a basic catalyst for transesterification reaction because it presents high activity, mild reaction conditions and lower cost and toxicity, compared to other commercial catalysts. The
\end{abstract}

effect of reaction time in the biodiesel yield has been investigated for biodiesel produced from soybean oil via methyl route in a batch reactor. In this process, methanol/soybean oil molar ratio was set at 12 , reaction temperature $65{ }^{\circ} \mathrm{C}$, catalyst/oil mass ratio $8 \%$ and reaction time of $1 \mathrm{~h}, 1.5 \mathrm{~h}$ and $2 \mathrm{~h}$. It was found that the reaction time has a great influence on the process. 
KEYWORDS: Biodiesel, Transesterification, Heterogeneous Catalyst.

\section{INTRODUÇÃO}

A consciência de uma tecnologia de produção limpa tem aumentando de forma global. A necessidade de uma alternativa aos combustíveis fósseis tem despertado o interesse de diversos pesquisadores ao longo dos últimos anos. Os combustíveis fósseis são fontes não renováveis de energia que geram poluentes e estão intimamente ligados ao aquecimento global, às mudanças climáticas e até mesmo a algumas doenças incuráveis. Os desafios iminentes e as implicações ambientais dos combustíveis fósseis têm sido amplamente divulgados na literatura (ARANSIOLA et al., 2014).

O biodiesel tem sido identificado como uma das opções para complementar os combustíveis convencionais. As suas vantagens sobre o diesel de petróleo não podem ser subestimadas: é um combustível seguro, renovável, não-tóxico e biodegradável; que não contém enxofre; e possui uma maior ação lubrificante. Além disso, seu uso gera numerosos benefícios sociais como a revitalização rural, criação de novos postos de trabalho e redução do aquecimento global (KISS, DIMIAN \& ROTHENBERG, 2008).

Segundo a Resolução $\mathrm{n}^{\circ} 7$ de 19 de março de 2008, Art. 2, da Agência Nacional do Petróleo, Gás Natural e Biocombustíveis, o biodiesel é definido como um combustível composto de alquil ésteres de ácidos graxos de cadeia longa, derivados de óleos vegetais ou de gorduras animais (ANP, 2008).

Esses materiais à base de ácidos graxos podem incluir diversos tipos de sementes como a soja, pinhão manso, dendê, conforme mostrado na Figura 1, além do uso de sebo, banha e óleo residuais (ARANSIOLA et al., 2010).

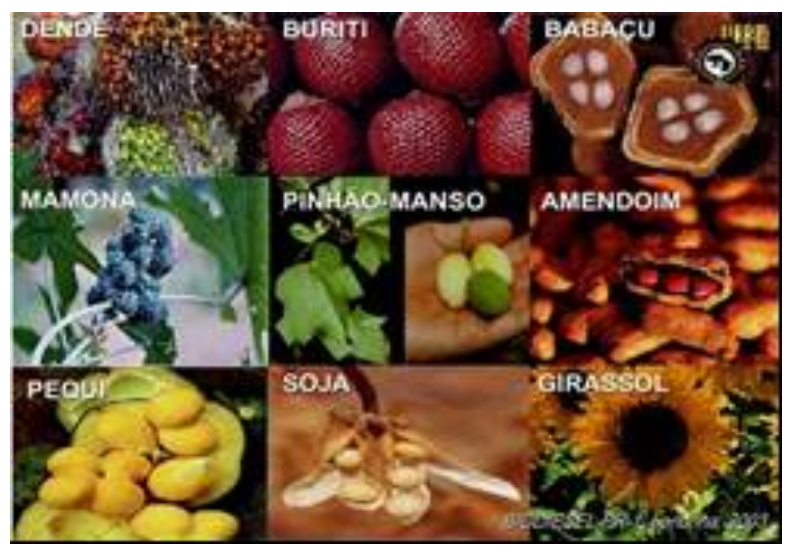

Figura 1: Variedade de oleaginosas (UNESP).

Os óleos vegetais mais comuns, cuja matéria-prima é abundante no Brasil, são soja, milho, algodão, babaçu e palma (PENILDO, 1981). O cultivo e a utilização da soja se originou na China há 
mais de 3000 anos atrás e posteriormente, se espalhou pelo Oriente (HOSKEN, 2003). Segundo o boletim mensal da ANP do mês de agosto do ano corrente, a soja representa cerca de $79 \%$ da produção de biodiesel no país, se tornando a principal matéria-prima utilizada no processo produtivo nacional (ANP, 2016).

O processo mais utilizado nos dias atuais é a transesterificação com catalisadores homogêneos em meio básico. A reação química ocorre entre triglicerídeos e álcool de cadeia curta, normalmente metanol ou etanol, em presença de catalisador (INTARAPONG et al., 2013).

O fato da reação ser conduzida por catálise homogênea torna o processo mais complexo devido às dificuldades durante a etapa de purificação do biodiesel. Portanto, muitos estudos indicam que os catalisadores heterogêneos apresentam grande capacidade de viabilizar a produção de biodiesel através de processos mais limpos e eficientes (DOMINGOS, 2010). Óxidos inorgânicos têm sido amplamente investigados como catalisadores para produção de biodiesel, sendo comum o uso de óxidos simples ou mistos, que podem ser obtidos pela calcinação de um sal na presença do óxido de interesse ou utilizando de fontes comerciais (CORDEIRO et al., 2011). Dentre esse grupo de catalisadores destaca-se o óxido de cálcio $(\mathrm{CaO})$ devido a sua elevada atividade em condições suaves de temperatura e pressão, por seu baixo custo e baixa toxicidade.

O objetivo desse trabalho visa estudar a influência da variável "tempo de reação" no rendimento em biodiesel através da reação de transesterificação metanólica do óleo de soja refinado em presença do catalisador heterogêneo óxido de cálcio ( $\mathrm{CaO})$.

\section{REVISÃO BIBLIOGRÁFICA}

Há uma série de fatores que influenciam o processo produtivo de biodiesel, os quais podem ser: concentração de catalisador, temperatura, velocidade de agitação, razão molar álcool/óleo e tempo reacional.

Kouzu et al. (2008) avaliaram os catalisadores de cálcio na forma de óxido (CaO), hidróxido $\left(\mathrm{Ca}(\mathrm{OH})_{2}\right)$ e carbonato $\left(\mathrm{CaCO}_{3}\right)$ na reação de transesterificação do óleo de soja refinado e óleo de cozinha processado, utilizando o metanol como solvente em excesso. Para efeitos de comparação foram estudados também os catalisadores de óxidos de metais alcalino terrosos $\mathrm{MgO}$ e SrO. Segundo os pesquisadores, o rendimento em ésteres metílicos na transesterificação do óleo de soja, indicou uma atividade catalítica na seguinte sequência: $\mathrm{MgO}<<\mathrm{CaO}<\mathrm{SrO}$. Ao empregar o $\mathrm{CaO}$ como catalisador heterogêneo na transesterificação do óleo de soja, foram obtidos rendimentos acima de $99 \%$ em ésteres metílicos, nas seguintes condições: 0,8\% em peso de $\mathrm{CaO}$, razão molar álcool/óleo de 12:1 e tempo de reação de 2 horas.

Liu et al. (2008) estudaram a utilização do CaO comercial na transesterificação do óleo de soja, sob condições ideais de temperatura a $65^{\circ} \mathrm{C}$, razão molar álcool/óleo de $12: 1$, concentração do catalisador de $8 \%$ em peso e adição de $2,03 \%$ de água em metanol. Após $3 \mathrm{~h}$ de reação, o rendimento em ésteres metílicos atingiu $95 \%$, resultado obtido por cromatografia gasosa.

Wu et al. (2013) estudaram a transesterificação do óleo de soja sob as condições de 12:1 para a razão molar metanol/óleo, temperatura de $65^{\circ} \mathrm{C}$ e $3 \%$ de catalisador heterogêneo (CaO suportado nas zeólitas NaY, KL e NaZSM-5, variando a concentração do óxido de cálcio de 5 a 50 
\%). Um rendimento de aproximadamente $65 \%$ em ésteres metílicos foi obtido após 2 horas de reação utilizando $30 \% \mathrm{CaO} / \mathrm{NaY}$, cujo resultado foi melhorado para $85 \%$ após 3 horas.

Tang et al. (2013) modificaram o óxido de cálcio com solução de bromooctano/hexano para uso como catalisador heterogêneo na transesterificação do óleo de soja, sob as seguintes condições operacionais: $65^{\circ} \mathrm{C}$ de temperatura; $15: 1$ de proporção molar entre metanol/óleo e 5 $\%$ de $\mathrm{CaO}$ modificado. O melhor rendimento em ésteres metílicos de ácidos graxos $(99,5 \%)$ foi obtido após 3 horas de reação, enquanto que o resultado após $2 \mathrm{~h}$ foi de $85 \%$.

Li et al. (2015) prepararam catalisadores oriundos da escória dos resíduos de carbonetos que se mostraram eficientes quando testados na produção de biodiesel a partir do óleo de soja com metanol. Foram investigados os efeitos da temperatura de calcinação do catalisador formado sobre o desempenho catalítico e, nas condições de calcinação de $650{ }^{\circ} \mathrm{C}$, o rendimento em ésteres metílicos foi de $91,3 \%$. Nesse caso, a reação de transesterificação ocorreu à uma temperatura de $65^{\circ} \mathrm{C}$, uma razão molar álcool:óleo de 9:1, com concentração de catalisador de $1,0 \%$ em peso e um tempo reacional de apenas $30 \mathrm{~min}$. O efeito do tempo de reação foi estudado e os resultados apresentados pelos autores indicam que após 15 min reacionais foi obtido um rendimento em ésteres metílicos de $85 \%$ e após um período de 45 min até 2 horas, o rendimento não apresentou significativas mudanças, oscilando na faixa de 90 a $92 \%$.

Portanto, o estudo do tempo de reação é importante para obtenção de maiores rendimentos em ésteres metílicos durante a produção de biodiesel.

\section{METODOLOGIA}

A seguir serão apresentados os procedimentos que foram seguidos para a execução dos experimentos em escala laboratorial.

\subsection{Materiais e equipamentos}

Os materiais utilizados nesse trabalho foram: óleo de soja refinado (Soya), catalisador CaO de alta pureza (Sigma-Aldrich) e solvente metanol PA (Neon).

Os equipamentos para montagem do sistema de batelada foram: balão de $250 \mathrm{~mL}$, agitador/aquecedor, condensador de refluxo, banho termostático e termômetro analógico.

Na Figura 2 encontra-se o esquema proposto para realização dos experimentos do tipo batelada. 


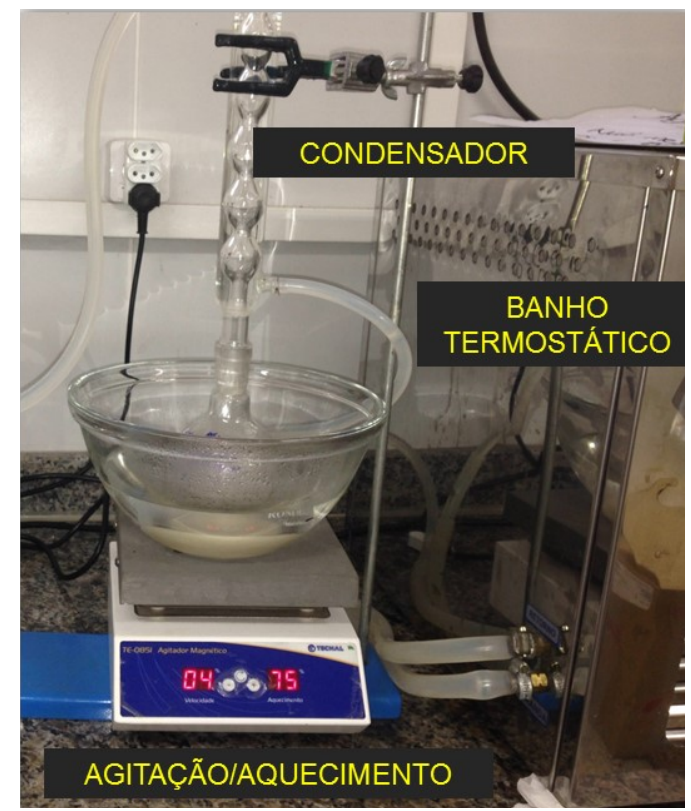

Figura 2: Processo esquemático para produção de biodiesel de soja.

\subsection{Ativação térmica do catalisador}

Inicialmente foi realizada uma ativação térmica do catalisador através de calcinação a $1000^{\circ} \mathrm{C}$ por $2 \mathrm{~h}$ a um fluxo de ar sintético de $100 \mathrm{~mL} / \mathrm{min}$ utilizando taxa de aquecimento de 10 ${ }^{\circ} \mathrm{C} / \mathrm{min}$, condições essas baseadas no estudo realizado por Reyero, Arzamendi \& Gandía (2014). 0 objetivo dessa ativação prévia é remover contaminantes de sua superfície pois carbonatos, hidróxidos e umidade levam à desativação antecipada do catalisador, visto que em contato com o ar ambiente esse material já apresenta elevada reatividade.

\subsection{Processo em batelada}

Nessa etapa a mistura contendo óleo, álcool e catalisador foi mantida sob agitação e aquecimento constante na temperatura de reação de $65^{\circ} \mathrm{C}$ variando os tempos reacionais em 1 h, 1,5 h e 2 h. As variáveis razão molar álcool/óleo de $12: 1$, temperatura de $65^{\circ} \mathrm{C}$ e concentração de catalisador de $8 \%$ em peso, foi baseada na literatura, através da pesquisa realizada por Liu et al. (2008).

Durante a reação, o álcool metílico foi evaporado continuamente devido à sua temperatura de ebulição ser próxima da temperatura reacional. Portanto, foi utilizado um condensador de refluxo tipo bola acoplado ao banho termostático, com objetivo de reaproveitar o metanol e manter constante a razão molar álcool/óleo, de forma a reduzir impactos negativos no rendimento em biodiesel.

Finalizada a reação, após os tempos pré-definidos, o catalisador foi separado da mistura através de filtração a vácuo a quente, e a glicerina foi separada do biodiesel através do processo de decantação por $24 \mathrm{~h}$. 
O catalisador foi lavado com metanol e hexano, conforme Li et al. (2015), para remover impurezas presentes em sua superfície e em seguida passou para etapa de secagem a $110{ }^{\circ} \mathrm{C}$ por 4h e posterior reativação por calcinação para reuso futuro.

O biodiesel produzido foi purificado por meio da evaporação do metanol empregando evaporador rotativo por $2 \mathrm{~h}$ a $100{ }^{\circ} \mathrm{C}$ e seco em estufa para remoção da umidade remanescente por $1 \mathrm{~h}$ a $104^{\circ} \mathrm{C}$.

\subsection{Caracterização físico-química do óleo}

A caracterização do óleo de soja utilizado na produção de biodiesel com o catalisador heterogêneo foi baseada nas normas da American Oil Chemists Society - AOCS, 1998. Desta forma, para a caracterização físico-química foram realizados os seguintes procedimentos:

\subsubsection{Determinação da acidez}

Utilizou-se o método AOCS Cd 3d-63 para determinação do índice de acidez da matériaprima, expressando o resultado em $\mathrm{mg} \mathrm{KOH/g}$ de óleo, referente ao teor de acidez presente no óleo de soja.

\subsubsection{Teor de umidade}

O equipamento utilizado para análise de umidade foi Karl Fischer Volumétrico, da marca Metler, modelo V-20 com reagentes de Karl Fischer Apura Combi Titrant 5, segundo a norma ASTM E203. O resultado foi expresso em porcentagem (\%) de teor de água, realizado pelo balanço de massa entre a amostra oleosa e o solvente utilizado (2-butanol).

\subsubsection{Determinação dos ácidos graxos livres}

Para determinação do teor de ácidos graxos livres utilizou-se o método de AOCS Ca 5a-40 onde o resultado foi expresso em \%.

\subsection{4 d. Determinação do índice de saponificáveis}

Para determinação desta propriedade utilizou-se o método AOCS Cd 3-25, cujo resultado foi expresso em mg KOH/g de óleo.

\subsection{Avaliação do rendimento em biodiesel}

O percentual de rendimento bruto em biodiesel foi determinado a partir da relação entre a massa de biodiesel produzida experimentalmente e a massa teórica determinada em função da massa molar do óleo de soja e da quantidade em massa do referido óleo alimentado no sistema, conforme mostra Equação (1), definida por Schuler (2010). 
$R_{\text {bruto }}=\frac{M M_{\text {óleo }} *}{m_{\text {óleo }}} \frac{m_{\text {biodiesel produzido }}}{3 * M M_{\text {biodiesel }}} * 100$

Onde:

MMóleo representa a massa molar do óleo de soja $(869,06 \mathrm{~g} / \mathrm{mol})$ (Schuler, 2010).

$\mathrm{MM}_{\text {biodiesel }}$ representa a massa molar do biodiesel de soja $(279,04 \mathrm{~g} / \mathrm{mol})($ Schuler, 2010).

$\mathrm{m}_{\text {óleo }}$ representa a massa do óleo de soja alimentado no processo batelada.

$m_{\text {biodiesel }}$ produzido representa a massa de biodiesel obtida experimentalmente.

\section{RESULTADOS E DISCUSSÕES}

Para um melhor entendimento dos resultados de rendimento em biodiesel, inicialmente foi necessário caracterizar a matéria-prima oleosa (óleo de soja refinado) a fim de determinar os parâmetros mínimos adequados para a produção de biodiesel.

A Tabela 1 apresenta as análises físico-químicas do óleo de soja com seus respectivos resultados, seguindo Freedman, Pryde e Mounts (1984) para os teores de ácidos graxos livres e umidade de referência, Tantirungrotechai, Thepwatee e Yoosuk (2013) para a acidez de referência e Anvisa (1999) para indicar a faixa de material saponificável permitida.

Tabela 1: Resultados das análises do óleo de soja.

\begin{tabular}{|c|c|c|}
\hline Análise & Resultado & Referência \\
\hline Ácidos graxos livres, \% & 0,125 & $<0,5^{(\mathrm{i})}$ \\
\hline Umidade, \% & 0,103 & $\leq 0,3^{(\mathrm{i})}$ \\
\hline Acidez, mg KOH/g & 0,091 & $<0,4^{\text {(ii) }}$ \\
\hline Saponificáveis, mg KOH/g & 191 & $189-195^{\text {(iii) }}$ \\
\hline
\end{tabular}

Diferentes valores de referência para material saponificável em óleo de soja refinado também são citados na literatura por Tantirungrotechai, Thepwatee \& Yoosuk (2013) $(185,6$ mg $\mathrm{KOH} / \mathrm{g}$ ) e por Li et al. (2015) (188,2 mgKOH/g).

Embora não exista uma especificação oficial para os óleos vegetais utilizados na produção de biodiesel, estudos revelaram que elevados índices de acidez e umidade, por exemplo, reduzem o rendimento para a formação de produtos desejados na reação (NASCIMENTO et al., 2009). 
Os resultados obtidos e mostrados na Tabela 1 apresentaram índice de acidez, teor de umidade, índice de ácidos graxos livres e de saponificação dentro das faixas consideradas ideais para produção de biodiesel.

A representação da separação das fases presentes na mistura após o processo de produção de biodiesel está mostrada na Figura 3.

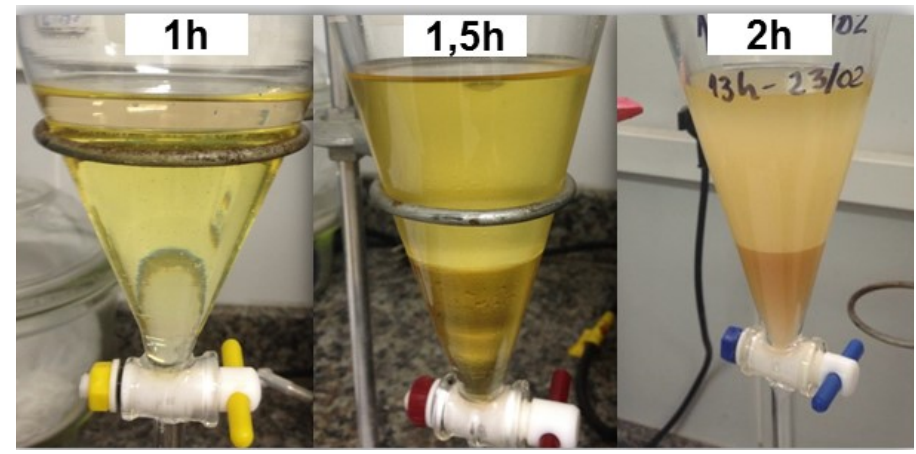

Figura 3: Separação de fases da mistura com diferentes tempos de reação

Conforme mostra a Figura 3, pode-se observar uma produção de biodiesel juntamente com a glicerina, co-produto da reação, a qual se apresenta na fase inferior da mistura devido à sua maior densidade.

A partir da Equação 1, foram obtidos os valores de rendimento em biodiesel para cada tempo reacional, como apresenta a Tabela 2.

Tabela 2: Resultados do rendimento bruto em biodiesel.

\begin{tabular}{c|c}
\hline Tempo de reação & Resultado \\
\hline $1 \mathrm{~h}$ & - \\
$1,5 \mathrm{~h}$ & $94,30 \%$ \\
$2 \mathrm{~h}$ & $95,03 \%$ \\
\hline
\end{tabular}

O ensaio realizado no tempo de $1 \mathrm{~h}$ indicou que a reação foi incompleta, dificultando a quantificação do biodiesel. Enquanto que para os tempos reacionais de 1,5 h e $2 \mathrm{~h}$ obtivemos rendimentos em biodiesel representativos.

\section{CONCLUSÕES}

Em função dos dados obtidos e análises físico-químicas realizadas, é possível concluir que a matéria-prima utilizada na síntese do biodiesel estava adequada às especificações ideais para o processo de transesterificação, influenciando assim em resultados finais satisfatórios. 
O biodiesel sintetizado com óleo de soja nas condições fixas de temperatura de $65{ }^{\circ} \mathrm{C}$, razão molar álcool/óleo de 12:1, 8 \% em peso de catalisador básico apresentou um maior rendimento ( 95\%) após duas horas de reação, quando comparado aos demais tempos avaliados neste trabalho.

Todavia, torna-se imprescindível a realização da análise cromatográfica afim de obter resultados mais precisos, pois os valores que foram encontrados são referentes ao rendimento em biodiesel bruto, o qual pode apresentar resquícios de óleo não reagido ou até mesmo metanol em excesso.

\section{REFERÊNCIAS}

ANP (2008). Resolução ANP No 7, DE 19.3.2008 - DOU 20.3.200. Recuperado em 10 de maio, 2016, do site http://www.udop.com.br/download/legislacao/comercializacao/juridico_legiscalcao/res_7_com ercializacao_biodiesel.pdf

ANP (2016). Boletim mensal do biodiesel (Agosto de 2016). Recuperado em 05 de setembro, 2016, do site http://www.anp.gov.br/SITE/acao/download/?id=82672.

AOCS (1998). Sampling and analysis of commercial fats and oils.

ANVISA (1999). Resolução RDC № 482, de 23/09/1999. Recuperado em 12 maio, 2016, do site http://www.anvisa.gov.br.

Aransiola, E. F., Betiku, E., Layokun, S. K., \& Solomon, B. O. (2010). Production of biodiesel by transesterification of refined soybean oil. International Journal of Biological and Chemical Sciences, 4(2), 391-399.

Aransiola, E. F., Ojumu, T. V., Oyekola, O. O., Madzimbamuto, T. F., \& Ikhu-Omoregbe, D. I. O. (2014). A review of current technology for biodiesel production: State of the art. Biomass and bioenergy, 61, 276-297.

Cordeiro, C. S., Silva, F. R., Wypych, F., \& Ramos, L. P. (2011). Catalisadores heterogêneos para a produção de monoésteres graxos (biodiesel). Química nova, 34(3), 477-486.

Domingos, A. K. (2010). Produção de biodiesel via catálise heterogênea (Tese de Doutorado. Universidade Federal do Rio de Janeiro, UFRJ. 2010. 181p).

Freedman, B., Pryde, E. H., \& Mounts, T. L. (1984). Variables affecting the yields of fatty esters from transesterified vegetable oils. Journal of the American Oil Chemists Society, 61(10), 16381643.

Hosken, B. (2003). Advances in soybean processing and utilization. In 12th Australian soybean conference proceedings.

Intarapong, P., langthanarat, S., Phanthong, P., Luengnaruemitchai, A., \& Jai-In, S. (2013). Activity and basic properties of $\mathrm{KOH} /$ mordenite for transesterification of palm oil. Journal of Energy Chemistry, 22(5), 690-700.

Kiss, A. A., Dimian, A. C., \& Rothenberg, G. (2007). Biodiesel by catalytic reactive distillation powered by metal oxides. Energy \& Fuels, 22(1), 598-604. 
Kouzu, M., Kasuno, T., Tajika, M., Sugimoto, Y., Yamanaka, S., \& Hidaka, J. (2008). Calcium oxide as a solid base catalyst for transesterification of soybean oil and its application to biodiesel production. Fuel, 87(12), 2798-2806.

Liu, X., He, H., Wang, Y., Zhu, S., \& Piao, X. (2008). Transesterification of soybean oil to biodiesel using $\mathrm{CaO}$ as a solid base catalyst. Fuel, 87(2), 216-221.

Li, F. J., Li, H. Q., Wang, L. G., \& Cao, Y. (2015). Waste carbide slag as a solid base catalyst for effective synthesis of biodiesel via transesterification of soybean oil with methanol. Fuel Processing Technology, 131, 421-429.

Nascimento, U. M., Vasconcelos, A. C. S., Azevedo, E. B., \& Silva, F. C. (2009). Otimização da produção de biodiesel a partir de óleo de coco babaçu com aquecimento por microondas. Eclética Química, 34(4), 37-48.

Penildo, P. F. (1980). O álcool combustível: obtenção e aplicação nos motores. Livraria Nobel.

Reyero, I., Arzamendi, G., \& Gandía, L. M. (2014). Heterogenization of the biodiesel synthesis catalysis: $\mathrm{CaO}$ and novel calcium compounds as transesterification catalysts. Chemical Engineering Research and Design, 92(8), 1519-1530.

Schuler, A. R. P. (2010). Processo de transesterificação metílica alcalina de óleos vegetais e gorduras animais e caracterização quantitativa de biodieseis. (Tese de Doutorado. Universidade Federal de Pernambuco, UFPE. 2010. 100p).

Tang, Y., Xu, J., Zhang, J., \& Lu, Y. (2013). Biodiesel production from vegetable oil by using modified $\mathrm{CaO}$ as solid basic catalysts. Journal of Cleaner Production, 42, 198-203.

Tantirungrotechai, J., Thepwatee, S., \& Yoosuk, B. (2013). Biodiesel synthesis over Sr/MgO solid base catalyst. Fuel, 106, 279-284.

UNESP (nd). Vigilantes da Energia - Biodíesel. Recuperado em 25 de maio, 2016, do site http://www.feg.unesp.br/emas/vigilantes/Fontes_de_Energia/biodiesel.htm.

Wu, H., Zhang, J., Wei, Q., Zheng, J., \& Zhang, J. (2013). Transesterification of soybean oil to biodiesel using zeolite supported $\mathrm{CaO}$ as strong base catalysts. Fuel Processing Technology, 109, 13-18. 